\title{
Primary nucleation of benzoic acid in aqueous
}

\section{ethanol solution}

Yan Zhao ${ }^{a, b}$, Guangyang Hou ${ }^{c}$, Vamsi Krishna Kamaraju, Yunliang He $^{b}$, Graham Power ${ }^{c}$, Brian Glennon ${ }^{b, c *}$

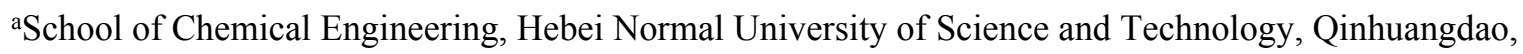

066004, PR China

bynthesis and Solid State Pharmaceutical Centre (SSPC), School of Chemical and Bioprocess Engineering,

University College Dublin, Dublin 4, Ireland

${ }^{\mathrm{c} A P C}$ Ltd, Dublin4, Ireland.

Corresponding author: brian.glennon@ucd.ie 


\section{Supporting Information}

Table S1 Solubility of benzoic acid in water/ethanol mixture

\begin{tabular}{cccc}
\hline$w_{\mathrm{w}}$ & & $x_{\mathrm{eq}}$ & \\
\hline $\mathrm{T} / \mathrm{K}$ & 294.15 & 298.15 & 303.15 \\
\hline 0.33 & $0.0797 \pm 2.3 \mathrm{E}-5$ & $0.0899 \pm 7.9 \mathrm{E}-5$ & $0.1042 \pm 0.0018$ \\
0.44 & $0.0492 \pm 0.0003$ & $0.0564 \pm 0.0001$ & $0.0664 \pm 0.0009$ \\
0.50 & $0.0353 \pm 0.0005$ & $0.0407 \pm 0.0009$ & $0.0502 \pm 0.0009$ \\
0.57 & $0.0226 \pm 1.5 \mathrm{E}-5$ & $0.0268 \pm 2.4 \mathrm{E}-5$ & $0.0338 \pm 0.0023$ \\
0.60 & $0.0163 \pm 5.4 \mathrm{E}-5$ & $0.0194 \pm 0.0005$ & $0.0262 \pm 0.0005$ \\
0.64 & $0.0107 \pm 3.2 \mathrm{E}-5$ & $0.0132 \pm 0.0001$ & $0.0180 \pm 0.0003$ \\
0.67 & $0.0053 \pm 4.9 \mathrm{E}-5$ & $0.0066 \pm 3.3 \mathrm{E}-5$ & $0.0099 \pm 0.0012$ \\
0.70 & $0.0040 \pm 2.7 \mathrm{E}-5$ & $0.0053 \pm 5.6 \mathrm{E}-5$ & $0.0099 \pm 0.0005$ \\
0.72 & $0.0036 \pm 2.8 \mathrm{E}-5$ & $0.0047 \pm 5.2 \mathrm{E}-5$ & $0.0070 \pm 0.0002$ \\
0.74 & $0.0026 \pm 1.9 \mathrm{E}-5$ & $0.0035 \pm 0.0001$ & $0.0051 \pm 1.1 \mathrm{E}-5$ \\
0.76 & $0.0021 \pm 3.0 \mathrm{E}-5$ & $0.0029 \pm 2.0 \mathrm{E}-5$ & $0.0038 \pm 4.1 \mathrm{E}-5$ \\
0.80 & $0.0012 \pm 0.0001$ & $0.0016 \pm 0.0002$ & $0.0019 \pm 0.0002$ \\
\hline
\end{tabular}


Table S2 The induction time with various supersaturation and temperature

\begin{tabular}{|c|c|c|c|c|c|c|}
\hline$x$ & $x_{\mathrm{eq}}$ & $w_{\mathrm{w}, \text { end }}$ & $\gamma$ & $a / a_{\mathrm{eq}}$ & $t_{\text {ind }}$ & $\mathrm{T} / \mathrm{K}$ \\
\hline 0.00434 & 0.00264 & 0.74 & 27.36 & 1.579 & $103 \pm 5$ & 294.15 \\
\hline 0.00446 & & & 27.28 & 1.619 & $53 \pm 4$ & \\
\hline 0.00459 & & & 27.13 & 1.659 & $20 \pm 2$ & \\
\hline 0.00473 & & & 27.10 & 1.706 & $12 \pm 2$ & \\
\hline 0.00491 & & & 26.99 & 1.761 & $6 \pm 1$ & \\
\hline 0.00538 & 0.00364 & 0.72 & 23.72 & 1.419 & $733 \pm 23$ & \\
\hline 0.00564 & & & 23.58 & 1.478 & $62 \pm 8$ & \\
\hline 0.00582 & & & 23.49 & 1.518 & $21 \pm 3$ & \\
\hline 0.00600 & & & 23.39 & 1.561 & $11 \pm 2$ & \\
\hline 0.00618 & & & 23.30 & 1.599 & $4 \pm 1$ & \\
\hline 0.00706 & 0.00530 & 0.67 & 20.01 & 1.288 & $240 \pm 25$ & \\
\hline 0.00712 & & & 19.98 & 1.298 & $100 \pm 9$ & \\
\hline 0.00719 & & & 19.95 & 1.308 & $40 \pm 3$ & \\
\hline 0.00729 & & & 19.91 & 1.325 & $15 \pm 4$ & \\
\hline 0.00740 & & & 19.86 & 1.341 & $6 \pm 2$ & \\
\hline 0.01859 & 0.01634 & 0.60 & 10.37 & 1.100 & $1469 \pm 5$ & \\
\hline 0.01907 & & & 10.31 & 1.117 & $48 \pm 3$ & \\
\hline 0.01913 & & & 10.26 & 1.123 & $23 \pm 2$ & \\
\hline 0.01928 & & & 10.24 & 1.129 & $11 \pm 2$ & \\
\hline 0.01954 & & & 10.18 & 1.139 & $5 \pm 1$ & \\
\hline 0.00491 & 0.00351 & 0.74 & 27.04 & 1.352 & $2038 \pm 48$ & 298.15 \\
\hline 0.00505 & & & 26.94 & 1.387 & $254 \pm 14$ & \\
\hline 0.00516 & & & 26.81 & 1.414 & $45 \pm 5$ & \\
\hline 0.00527 & & & 26.73 & 1.441 & $21 \pm 3$ & \\
\hline 0.00533 & & & 26.64 & 1.453 & $12 \pm 1$ & \\
\hline 0.00633 & 0.00465 & 0.72 & 23.28 & 1.310 & $1548 \pm 50$ & \\
\hline 0.00640 & & & 23.24 & 1.322 & $480 \pm 24$ & \\
\hline 0.00657 & & & 23.15 & 1.351 & $80 \pm 13$ & \\
\hline 0.00676 & & & 23.05 & 1.385 & $20 \pm 6$ & \\
\hline 0.00686 & & & 23.00 & 1.402 & $10 \pm 2$ & \\
\hline 0.00696 & & & 22.95 & 1.419 & $4 \pm 1$ & \\
\hline 0.00870 & 0.00666 & 0.67 & 19.40 & 1.251 & $168 \pm 9$ & \\
\hline 0.00875 & & & 19.38 & 1.257 & $65 \pm 7$ & \\
\hline 0.00880 & & & 19.36 & 1.264 & $40 \pm 4$ & \\
\hline
\end{tabular}




\begin{tabular}{|c|c|c|c|c|c|c|}
\hline$x$ & $x_{\mathrm{eq}}$ & $w_{\mathrm{w}, \text { end }}$ & $\gamma$ & $a / a_{\mathrm{eq}}$ & $\mathrm{t}_{\text {ind }}$ & $\mathrm{T} / \mathrm{K}$ \\
\hline 0.00884 & & & 19.34 & 1.268 & $31 \pm 2$ & 298.15 \\
\hline 0.00891 & & & 19.31 & 1.276 & $15 \pm 2$ & \\
\hline 0.00897 & & & 19.29 & 1.283 & $8 \pm 2$ & \\
\hline 0.02227 & 0.01945 & 0.60 & 9.90 & 1.106 & $1145 \pm 27$ & \\
\hline 0.02287 & & & 9.88 & 1.118 & $246 \pm 15$ & \\
\hline 0.02306 & & & 9.85 & 1.124 & $120 \pm 10$ & \\
\hline 0.02344 & & & 9.80 & 1.136 & $32 \pm 3$ & \\
\hline 0.02424 & & & 9.68 & 1.162 & $5 \pm 1$ & \\
\hline 0.00741 & 0.00508 & 0.74 & 25.53 & 1.379 & $242 \pm 20$ & 303.15 \\
\hline 0.00756 & & & 25.43 & 1.405 & $98 \pm 8$ & \\
\hline 0.00765 & & & 25.38 & 1.418 & $80 \pm 7$ & \\
\hline 0.00781 & & & 25.29 & 1.440 & $42 \pm 5$ & \\
\hline 0.00790 & & & 25.23 & 1.455 & $22 \pm 4$ & \\
\hline 0.00807 & & & 25.13 & 1.481 & $9 \pm 2$ & \\
\hline 0.00899 & 0.00702 & 0.72 & 22.01 & 1.224 & $3 \hat{5} 2 \overline{2} \pm 32$ & \\
\hline 0.00917 & & & 21.92 & 1.245 & $54 \pm 11$ & \\
\hline 0.00929 & & & 21.86 & 1.257 & $21 \pm 4$ & \\
\hline 0.00938 & & & 21.81 & 1.268 & $12 \pm 3$ & \\
\hline 0.00948 & & & 21.77 & 1.280 & $4 \pm 1$ & \\
\hline 0.01150 & 0.00990 & 0.67 & 18.39 & 1.124 & $842 \pm 9$ & \\
\hline 0.01165 & & & 18.33 & 1.135 & $180 \pm 7$ & \\
\hline 0.01181 & & & 18.28 & 1.147 & $87 \pm 4$ & \\
\hline 0.01212 & & & 18.17 & 1.169 & $24 \pm 2$ & \\
\hline 0.01253 & & & 18.02 & 1.197 & $8 \pm 2$ & \\
\hline 0.02987 & 0.02616 & 0.60 & 9.01 & 1.084 & $154 \pm 12$ & \\
\hline 0.02995 & & & 9.00 & 1.086 & $116 \pm 10$ & \\
\hline 0.03015 & & & 8.98 & 1.090 & $87 \pm 7$ & \\
\hline 0.03086 & & & 8.89 & 1.105 & $25 \pm 5$ & \\
\hline 0.03155 & & & 8.81 & 1.119 & $10 \pm 3$ & \\
\hline
\end{tabular}



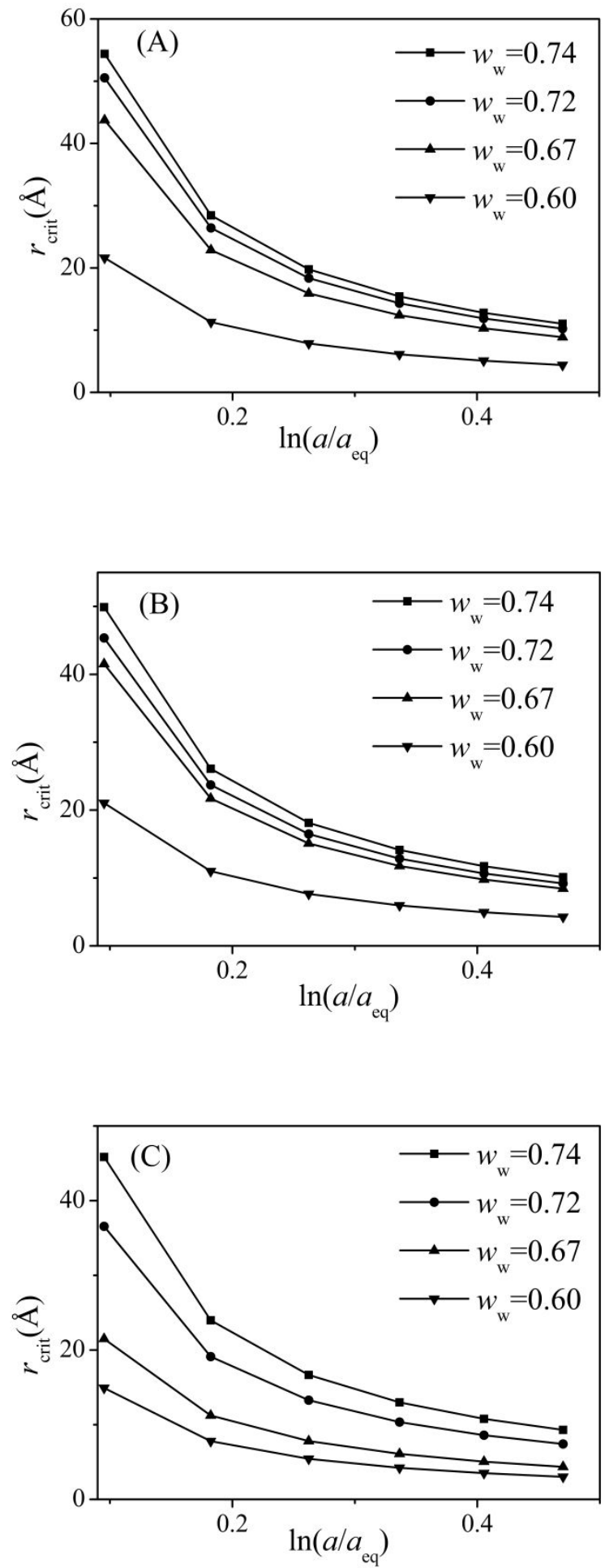

Figure S1 Plot of radius of critical nucleus, $r_{\text {crit }}(\AA)$, with driving force, $\ln \left(a / a_{\mathrm{eq}}\right)$, at different mass fraction of water $\left(w_{\mathrm{w}}\right)$ : (A) $\mathrm{T}=294.15 \mathrm{~K}$; (B) $\mathrm{T}=298.15 \mathrm{~K}$; (C) $\mathrm{T}=303.15 \mathrm{~K}$ 

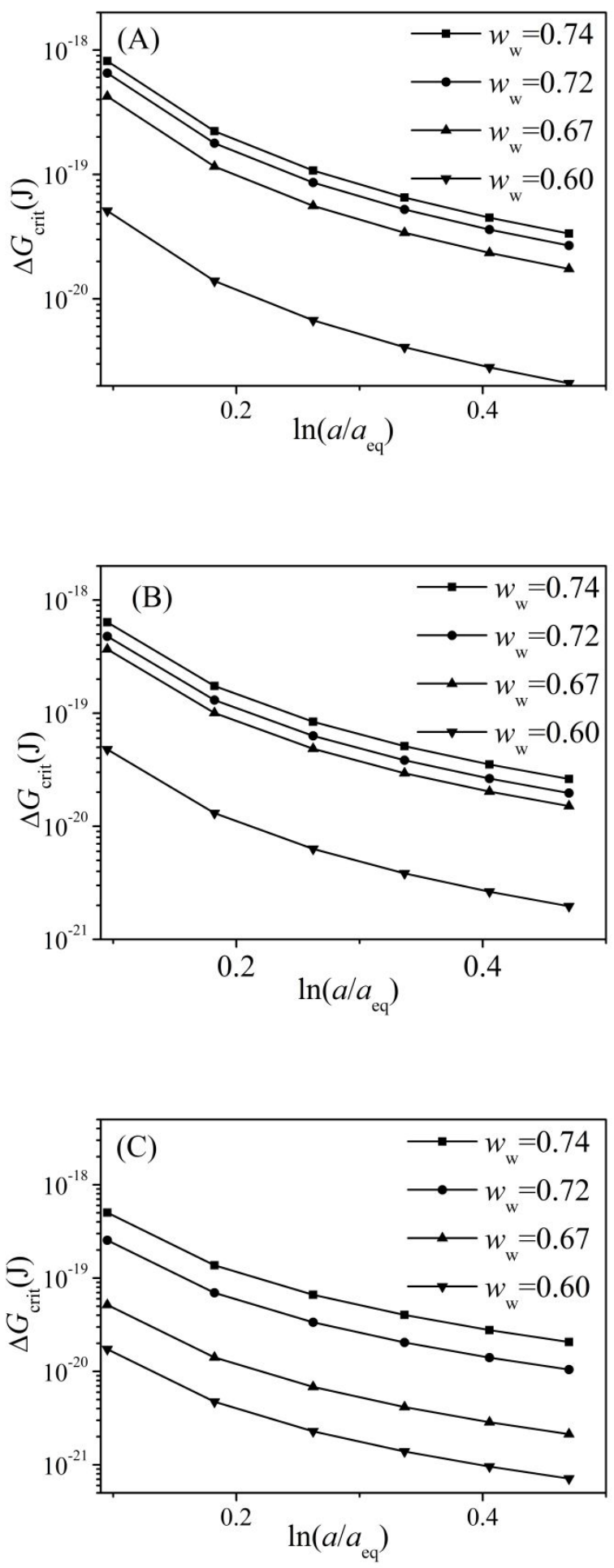

Figure S2 Plot of activation energy for nucleation, $\Delta G_{\text {crit }}(J)$, with driving force, $\ln \left(a / a_{\mathrm{eq}}\right)$, at different mass fraction of water ( $\left.w_{\mathrm{w}}\right)$ : (A) $\mathrm{T}=294.15 \mathrm{~K}$; (B) $\mathrm{T}=298.15 \mathrm{~K}$; (C) $\mathrm{T}=303.15 \mathrm{~K}$ 